\title{
Phase separation of multiphase droplets in a digital microfluidic device
}

\author{
Mun Mun Nahar and Hyejin Moon* (1)
}

\begin{abstract}
This study reports the first comprehensive investigation of separation of the immiscible phases of multiphase droplets in digital microfluidics (DMF) platform. Electrowetting-on-dielectric (EWOD) actuation has been used to mechanically separate the phases. Phase separation performance in terms of percentage residue of one phase into another phase has been quantified. It was conceived that the residue formation can be controlled by controlling the deformation of the phases. The larger capillary number of the neck forming phase is associated with the larger amount of deformation as well as more residue. In this study, we propose two different ways to control the deformation of the phases. In the first method, we applied different EWOD operation voltages on two phases to maintain equal capillary numbers during phase separation. In the second method, while keeping the applied voltages same on both sides, we tested the phase separation performance by varying the actuation schemes. Less than $2 \%$ of residue was achieved by both methods, which is almost $90 \%$ improvement compared to the phase separation by the conventional droplet splitting technique in EWOD DMF platform, where the residue percentage can go up to 20\%.
\end{abstract}

Keywords: Electrowetting-on-dielectric, Digital microfluidics, Multiphase droplet, Compound droplet, Phase separation, Droplet splitting

\section{Introduction}

In recent years, solvent extraction and partitioning processes have been miniaturized into lab-on-a-chip devices due to their favorable position in terms of better mass transfer efficiency, higher specific interfacial area, and the lower consumption of reagents compared to classical macroscale approaches [1]. Liquid-liquid micro extraction processes have been performed for separation and partitioning of cells, proteins, molecules, and ions in various applications [2-8]. These processes utilize the interface between two immiscible liquids. Typically, one is aqueous phase and the other often comprise of organic solvent, another immiscible aqueous phase (e.g. aqueous two-phase system), oil or ionic liquid. Following the solvent extraction, the two immiscible phases are separated in order to retrieve the extracted substance. Until now, phase separation has been achieved by adding surfactant [9], by using structured microchannels

*Correspondence: hyejin.moon@uta.edu

Mechanical and Aerospace Engineering Department, University of Texas at Arlington, 500 W. First Street, Arlington, TX 76019, USA with differing wettability [10], by surface treatments $[8,11-13]$ or by combining several techniques $[14,15]$. Most of these methods utilize laminar parallel flows in microchannels. A laminar flow helps to maintain a stable interface between two liquids, however, it limits the interfacial area, thus hinders mass transfer rate, which poses the major challenge in these technologies. Instead of a laminar parallel flow, multiphase (e.g., double emulsion) droplet flows in a microchannel can be used and phase separation can be achieved by breakup of a multiphase droplet [16-23]. Since these technologies involve droplets in continuous micro channel environments or in a linear or extensional flow field in a viscous medium, the knowledge gained from these studies is not transferrable into electrowetting on dielectric (EWOD) digital microfluidic (DMF) platform. Compared to microchannels, EWOD DMF offers various advantages such as high flexibility, reconfigurability, and freedom from moving parts such as pumps, valves, tubing, etc. [24-26]. Wijethunga et al. [27] used EWOD DMF to combine the two immiscible liquid phases to form a droplet before extraction and to separate the phases after extraction. Fan et al. 
[28] demonstrated phase separation using EWOD splitting technique. But none of these studies addressed the issue of the partial recovery of the extracted material due to incomplete separation of the phases which is an inherent issue of multiphase droplet splitting. In order to transform EWOD DMF into a suitable candidate for many of the applications involving multiphase droplets, a robust phase separation technique needs to be established exclusively.

This present study is the first one to focus explicitly on the mechanism of phase separation of a multiphase droplet. Additionally, we aim to suggest some technical guidelines to achieve better separation efficiency with minimal residue.

\section{Theoretical backgrounds}

Thermodynamically, multiphase droplets prefer to remain at the phase equilibrium state with the minimum free energy configuration. This means that the state of two separated droplets is only favored when both liquids have smaller interfacial tensions than the third surrounding medium [22, 23]. Therefore, core-shell or partially engulfed multiphase droplets do not readily separate phases and they require additional force to do so. In an EWOD DMF, additional capillary forces such as wetting and de-wetting forces can be exerted on multiphase droplets so that they may separate their phases.

Before looking into phase separation process, let's revisit droplet splitting process in an EWOD DMF device [29-31] where a droplet is sandwiched between two parallel plates. The mechanism of droplet splitting is shown in Fig. 1.

The droplet is initially positioned in such a way that it covers two activated electrodes (Fig. 1a(i)). Due to initial wetting, the droplet takes the shape of two central electrodes and remains at equilibrium. Then two side electrodes next to the droplet are switched on while the central electrodes are switched off. As the droplet meniscus sees the activated electrodes, it is pulled toward them with a net wetting force acting on the longitudinal direction. As a result, the droplet is stretched in that direction. As more liquid flows towards the active electrodes, the amount of liquid at the middle keeps decreasing due to mass conservation. Simultaneously, on the middle electrodes surface, the contact angle of the liquid restores to hydrophobic state and the droplet starts to de-wet the surface (Fig. 1a(ii)). This results in pulling of the droplet meniscus inward (i.e., transversely) and the middle part of the droplet becomes narrower. The narrowing of the droplet is called "necking". Necking continues until droplet menisci meet each other so that the neck breaks at the pinch-off point (Fig. 1a(iii)) and the single droplet splits into two daughter droplets. Typically, with the same voltage application at both sides, a single-phase droplet splitting is a symmetric process such that two daughter droplets have approximately less than $1 \%$ volume differences [30]. This volume difference is due to hydrodynamic (e.g., Rayleigh-Taylor) instability which may be induced by the surface roughness or other random irregularities on the interface. While there are many reports regarding droplet splitting in DMF [29-32], a few of them discuss about asymmetric splitting [30, 31].

In principle, phase separation can be performed by conventional droplet splitting techniques including EWOD as shown in Fig. 1b. When wetting forces are applied to two sides, the droplet is longitudinally stretched and transverse necking starts (Fig. 1b(ii)). But the necking location and the interface position do not align with each other. Therefore, after pinching-off, some a

(i)

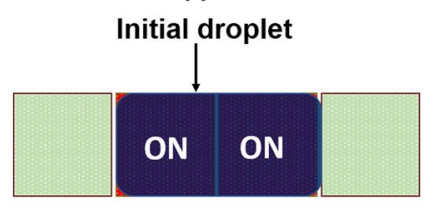

b

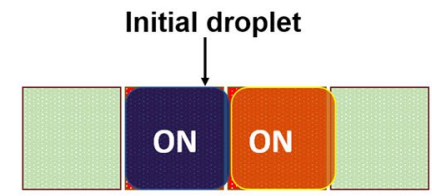

(ii)

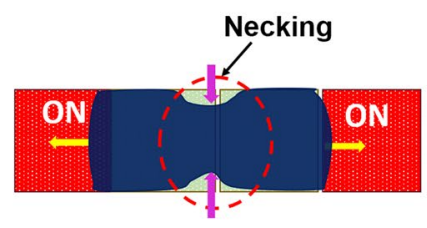

(ii)

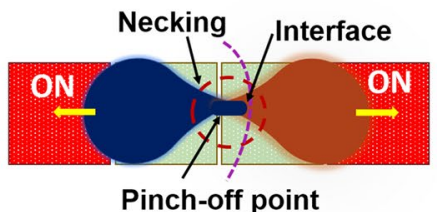

(iii)

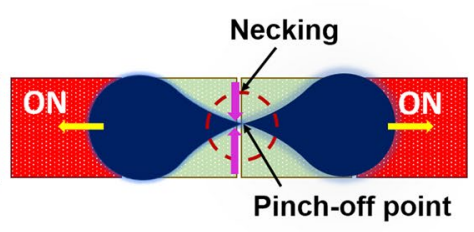

(iii)

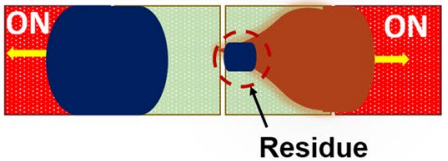

Fig. 1 Steps during splitting of: a a single-phase droplet, and $\mathbf{b}$ a multiphase droplet for phase separation. Illustrations are top views 
residue is left in one of the phases (Fig. 1b(iii)). Unlike single-phase droplet splitting, phase separation is inherently an asymmetric process. All the factors causing unequal splitting are deduced into one observable effect: unequal deformations of two phases. Therefore, in this study, we intended to control deformation using two different strategies to reduce the asymmetry in phase separation in order to control the amount of residue. This is discussed below:

\section{i. By keeping equal capillary numbers}

Capillary number $(C a=\mu U / \sigma)$ is a dimensionless number that estimates the relative strength of the surface tension force and viscous force. Here, $\sigma$ is the surface tension, $\mu$ is the dynamic viscosity and $U$ is the velocity of a fluid. In microfluidics, capillary number is often used to estimate the extent of deformation of fluid segments. A high capillary number is related to a large amount of deformation and vice versa. During splitting, necking is necessary which means that the droplet has to be deformed. Now if both sides of the droplet deform similarly, meaning that the profile of the droplet footprint on both sides of the neck is similar, splitting happens to be almost symmetric. By keeping capillary numbers equal on both sides, the same extent of deformations in both sides are expected. Many previous studies tried to estimate the droplet velocity in EWOD DMF [33-36]. Especially, Baird et al. [33] proposed a simplified model to approximate droplet steady-state velocity by neglecting viscous drag from the filler medium and the contact line friction. According to this formulation, droplet velocity during EWOD actuation is given as follows:

$$
\mathrm{U}=C \frac{V^{2} h}{24 \mu L}
$$

where $\mathrm{C}$ is the system capacitance (mostly provided by dielectric layers), $\mathrm{V}$ is the applied voltage, $\mathrm{h}$ is the channel height (i.e., the gap between two parallel plates), and L is the characteristic length of the system. Substituting fluid velocity into capillary number gives:

$$
C a=C \frac{V^{2} h}{24 \sigma L}
$$

Now, if we have two fluids, namely fluid 1 and 2 forming a multiphase droplet undergoing separation process, their capillary numbers should be in the same range to keep their deformation close to each other. In other words, the following condition:

$$
\frac{C a_{1}}{C a_{2}}=\frac{\left(V_{1} / V_{2}\right)^{2}}{\left(\sigma_{1 / \sigma_{2}}\right)} \approx 1
$$

should be maintained. Note that since both fluids run in the same device, $\mathrm{C}, \mathrm{h}$, and $\mathrm{L}$ are same for both fluids. Therefore,

$$
\left(\frac{V_{1}}{V_{2}}\right)^{2}=\frac{\sigma_{1}}{\sigma_{2}}
$$

This means that for a given multiphase droplet, to keep capillary numbers of both phases equal, unequal voltages should be applied depending on the ratio of their surface tensions. For example, for a water-ionic liquid multiphase droplet, applied voltage on water side should be 1.2 times higher than that on the ionic liquid side $\left(\frac{V_{1}}{V_{2}}=\sqrt{ }\left(\frac{.072}{.05}\right)\right)$.

\section{ii. By directly controlling the deformation}

Another way to obtain symmetric splitting of multiphase droplet is by directly controlling the deformation of both phases so that their morphologies remain equal in spite of having different fluid properties. We discussed droplet deformation in our previous publication [36]. As shown in Fig. 2, during a droplet transition from a deactivated electrode to adjacent activated one, the droplet deforms because of the interplay between two oppositely directed forces [i.e., longitudinal wetting forces $\left(f_{e w}\right)$ and transverse de-wetting forces $\left(f_{\text {dew }}\right)$ ]. Therefore, by minimizing the overlap of droplet footprint with any deactivated electrode, deformation can be minimized. For square electrodes (Fig. 2a), where a droplet has to travel a distance equal to one electrode width in one sequence, the droplet has the freedom to deform within that distance. By shortening the electrode width (Fig. 2b), the freedom is restricted and the droplet meniscus is confined within the electrode boundary at each sequence. Therefore, the droplet is forced to experience less deformation [36]. So, we hypothesize that using the slender shape of electrodes in a manner that each phase is confined equally will achieve symmetry in separation. This is a unique feature of EWOD system where deformation can be controlled in a digitized manner, which is impossible to achieve in continuous microfluidics. Based on our hypothesis, we are proposing different actuation schemes which will be discussed in detail in upcoming sections.

\section{Experimentals}

\section{Device fabrication}

All EWOD devices were fabricated in Shimadzu Institute Nanotechnology Research Center at The University of Texas at Arlington. Device fabrication steps are shown in Fig. 3. Conductive layer (either indium tin oxide or chromium) coated on glass substrates was patterned by wet etching and dielectric layer (SU-8, $5 \mu \mathrm{m})$ and hydrophobic layer (Teflon, $300 \mathrm{~nm}$ ) were spin-coated on top 

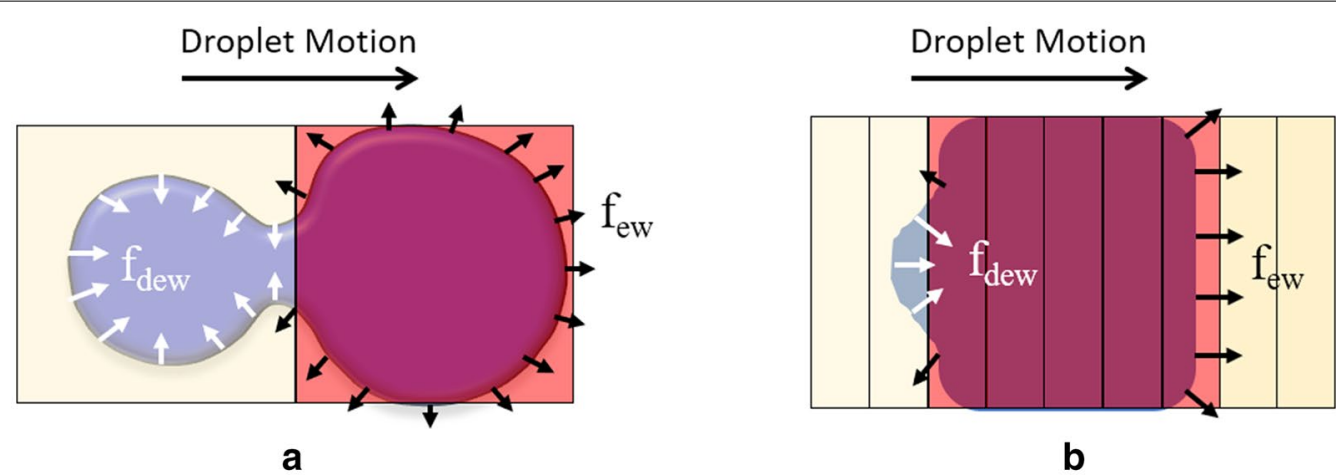

b

Fig. 2 A droplet during transition from a grounded electrode to an activated one (top view). Activated electrode is indicated in red. Electrowetting force, $f_{\text {ew }}$ (black arrows) and dewetting force, $f_{\text {dew }}$ (white arrows), on the triple point contact line are shown. a Droplet transition on square shape electrodes. b Droplet transition on rectangular electrodes with short width

and oven baked. The detail description of the fabrication steps can be found in Additional file 1: S1. Slender shape electrodes were fabricated [36] to perform separation tests. L-junction reservoir designs were used to ensure accuracy and consistency in droplet generation [30].

\section{Materials and device operation conditions}

To form a multiphase droplet, two immiscible liquidswater and ionic liquid (1-butyl-3-methylimidazolium hexafluorophosphate ([bmim][PF6])) were used. The gap between top and bottom plates of devices was kept at $100 \mu \mathrm{m}$ for all cases. A voltage of $110 \mathrm{~V}$ and/or $130 \mathrm{~V}$ at $1 \mathrm{kHz}$ frequency were applied. To provide different voltages, two voltage generators with separate amplifiers were used.

\section{Test steps}

A partially engulfed multiphase droplet was formed by merging a water droplet with an ionic liquid (IL) droplet. Two separate reservoirs were used for generating the droplets as shown in Fig. 4a. After generation, each droplet was taken to the middle portion of the device to merge with the other droplet (Fig. 4b, c). Multiphase droplets were spontaneously created and remained there until the next operation. Different actuation schemes were used to separate phases of a multiphase droplet. Details of actuation schemes are described in "Results and discussions" section.

\section{Data acquisition and analysis}

During the tests, videos of droplet motion were recorded using Hirox KH-1300 digital microscope system. Later, images were extracted from videos and the footprint area and the velocity of each phase were measured using ImageJ software (NIH). The volume of each phase was estimated by multiplying the footprint area by the height of the channel. Residue volume is calculated as a percentage of the volume of the second phase in the primary phase after the phase separation.
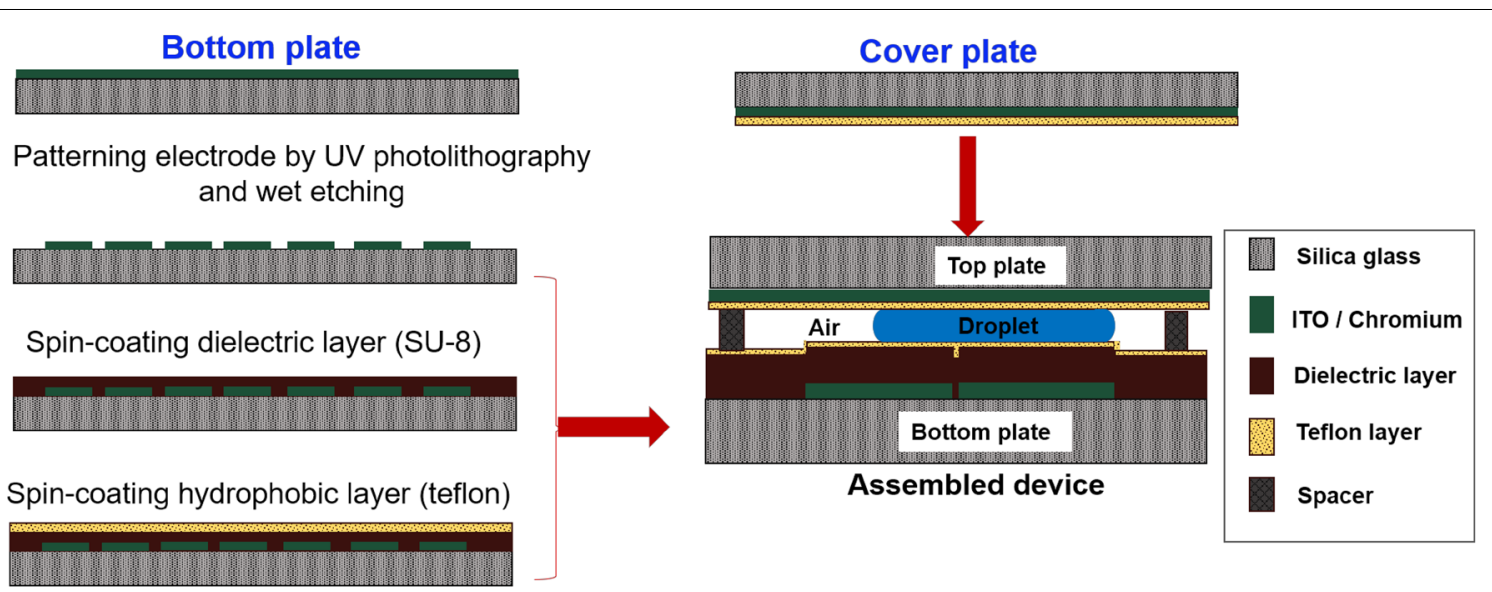

Fig. 3 Fabrication process of an EWOD DMF device (side view) 


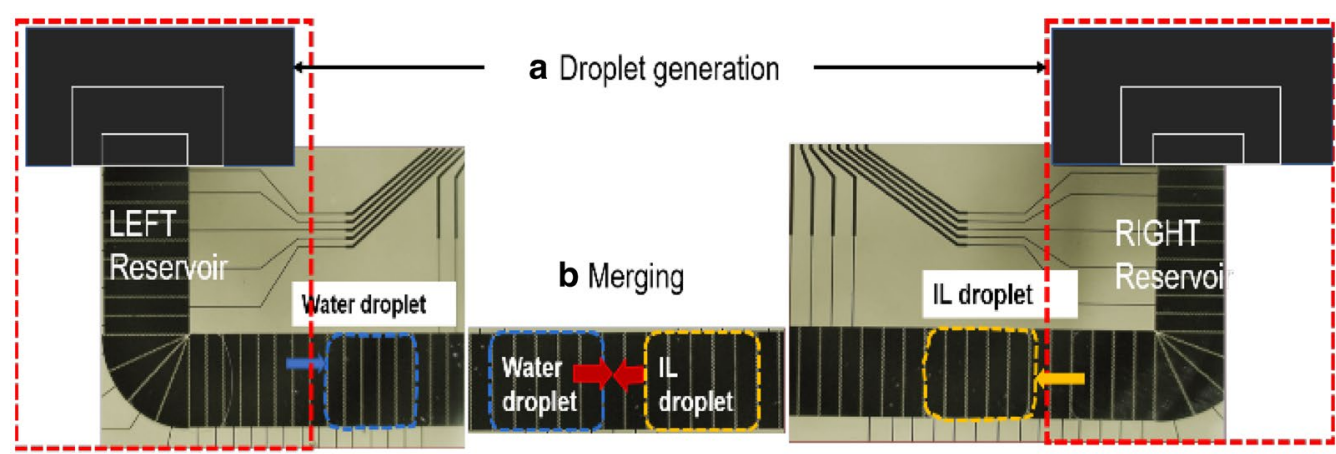

c Multiphase droplet

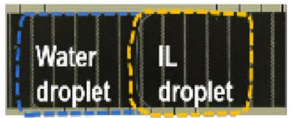

Fig. 4 Steps of an experiment: a generation of water and ionic liquid (IL) droplets from each reservoir, $\mathbf{b}$ merging the generated droplets, and $\mathbf{c}$ the multiphase droplet formed

\section{Results and discussions}

Tests were performed addressing the two hypotheses regarding controlling deformation that we derived in "Theoretical backgrounds" section. First, different voltages were applied to achieve equal capillary numbers for both phases. Second, various splitting schemes in order to directly control deformations were introduced and tested.

\section{Case 1: Different applied voltages}

In this case study, EWOD device with conventional square shape electrodes were used and tests were run to compare the effect of applied voltages. Phase separation was performed by pulling a multiphase droplet from opposite directions as voltages were applied to two side electrodes next to the droplet. When the same voltages were applied, the front of IL phase moved three times faster than that of water because of differences in properties of the two fluids. Therefore, IL deformed more than water during transition as predicted by its higher capillary number $\left(\mathrm{Ca}_{\text {water }} \sim 10^{-4}\right.$ to $10^{-6}$ and $\mathrm{Ca}_{\mathrm{IL}} \sim 10^{-2}$ to $\left.10^{-3}\right)$. As seen in Fig. $5 \mathrm{a}(\mathrm{i}$ and ii), compared to the water phase, IL phase responded faster to the applied force and proceeds quickly towards the active electrode (to right). In the meantime, the water phase remained almost still with negligible deformation. Within a very short period, the IL phase deformed enough to form a neck (Fig. 5a(iii)). All the while the water phase remained unmoved. The neck kept thinning because of the pulling force and finally pinched off at an off-center position away from the interface. The multiphase droplet was broken into two parts with a large amount of IL residue on the water phase (Fig. 5a(iv)).
Interestingly, when the process was repeated with different applied voltages on two sides, we observed a different scenario. Voltages of $130 \mathrm{~V}$ and $110 \mathrm{~V}$ were applied on water and IL side, respectively. As seen in Fig. $4 \mathrm{~b}(\mathrm{i}$ and ii), both water and IL phases were moving at similar paces and their profiles were quite similar, which means they deformed in the same manner. Consequently, the amount of IL residue in the water phase was significantly less as shown in Fig. 5b(iv).

Percentage residue is plotted in Fig. 6. Each column shows the average from at least 4 tests. In each test, newly generated water and IL droplets were merged to form a fresh multiphase droplet. It is obvious from the figure that, when we applied the same voltage, the phase separation performance was poor as an average of approximately $17 \%$ residue of IL was left on the water. The performance improved greatly by applying unequal voltages to phases as the residue amount reduced to $2.37 \%$.

\section{Case 2: Actuation schemes}

In this case study, the device was operated with a single voltage $(110 \mathrm{~V})$ to compare the effect of actuations schemes. Figure 7 lists actuation schemes tested in this study. Conventionally, droplet spitting has been performed by using square shaped electrodes and transition distances are always even multiples of the dimension of one square electrode. So, we consider this our reference and term it as "SQ" scheme (Fig. 7a). For other schemes, slender shape electrodes were used. Here, the width of a slender electrode is one fifth of the width of a square electrode. Schemes using slender electrodes are termed "SL" (Fig. 7b, c). In the beginning of SL1 scheme, two electrodes in the middle 


\section{a Same voltage}

(i)

(ii)

(iii)

(iv)

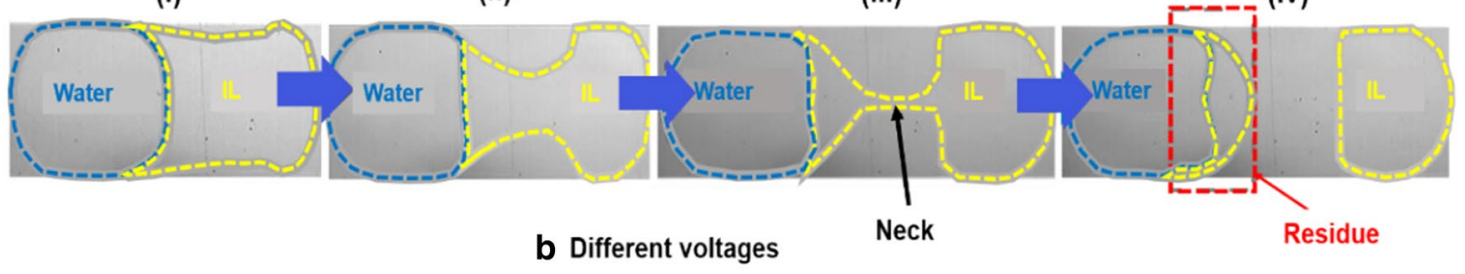

(i)

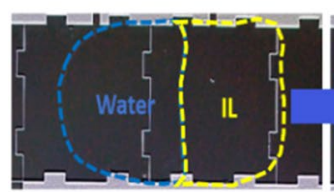

(ii)
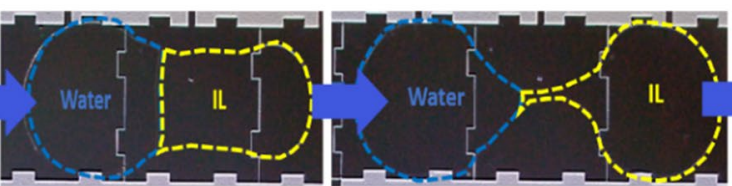

(iv)

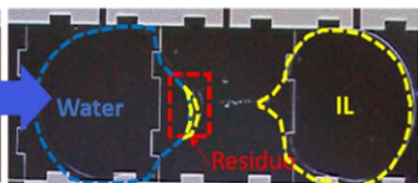

Fig. 5 Droplet profiles at different stage during phase separation using: a same voltage of $110 \mathrm{~V}$ on both phases, and $\mathbf{b} 130 \mathrm{~V}$ on water and $110 \mathrm{~V}$ on ionic liquid, respectively

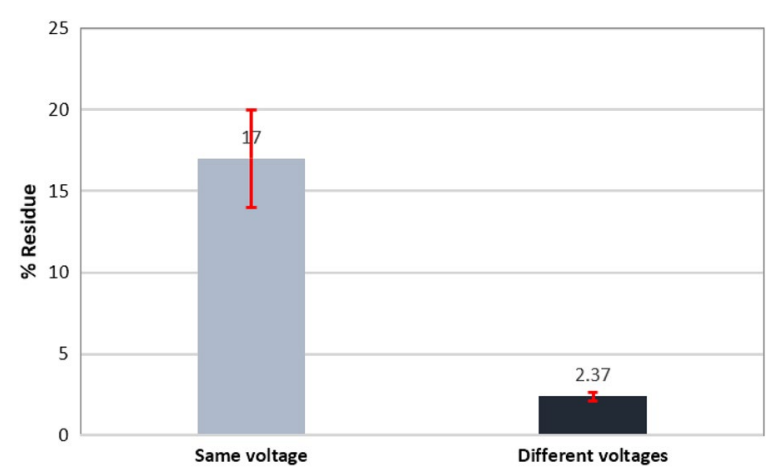

Fig. 6 Percent residue comparison for cases of same applied voltage and the different applied voltages. Error bars represent standard deviations

of the droplet were turned off while one electrode on each side of the droplet were turned on. In next step, two additional electrodes in the middle were turned off and one at each side were turned on. We defined SL2 schemes where four electrodes were turned off in the middle and two electrodes are turned on each side in each sequence. Sequences repeated until the droplet pinch-off occurs.

The use of slender electrode actuation highly constrains the droplet morphology [36] during transition regardless of difference in velocities of phases, as well as unequal capillary numbers. As seen in Fig. 8a, SQ scheme allowed both phases to elongate over a long distance in one sequence so that the chance for hydrodynamic instability to play a role in asymmetric positioning of the "separation point" was high. On the other hand, due to the smaller transition distance of SL1 scheme (20\% of SQ scheme), the morphology of each phase was constrained by the boundary of activated electrodes. Therefore, deformations of both phases were more symmetrical regardless of differences in velocities and capillary numbers. The effects are obvious in Fig. 8b for SL1. The amount of residue was significantly less compared to the case of SQ scheme. As expected from the above reason, the result of SL2 scheme test (Fig. 8c) presents a residue amount smaller than that of SQ. The experimental video of separation using SL1 and SQ schemes are presented in additional file 2 and 3, respectively. It is evident that the different schemes were able to provide ways to vary deformation.

From this speculation, we introduced another type of schemes where one phase was kept still in its initial position so that its meniscus was forced to conform to the electrode shape and deformation was minimized. As shown in Fig. $7 d-g$, four additional schemes-IL fixedSQ, IL fixed-SL1, Water fixed-SQ and Water fixed-SL1were tested.

When both phases moved, IL was the more deforming phase. However, when IL was fixed at the initial position by EWOD force (Fig. 9a), it deforms less than water phase. As the water phase moved further, the neck occurred and pinched off in the water phase as leaving a little water residue in the IL phase. On the other hand, when water was fixed (Fig. 9b), IL deformed significantly as it moved, and it left huge residue in the water phase after the pinch-off. While IL-fixed scheme improved separation performance significantly in $\mathrm{SQ}$, it did not make much difference in SL1. Unlike the simple SL1 (Fig. 8b), water deformed more in IL-fixed SL1 (Fig. 9c), however, 


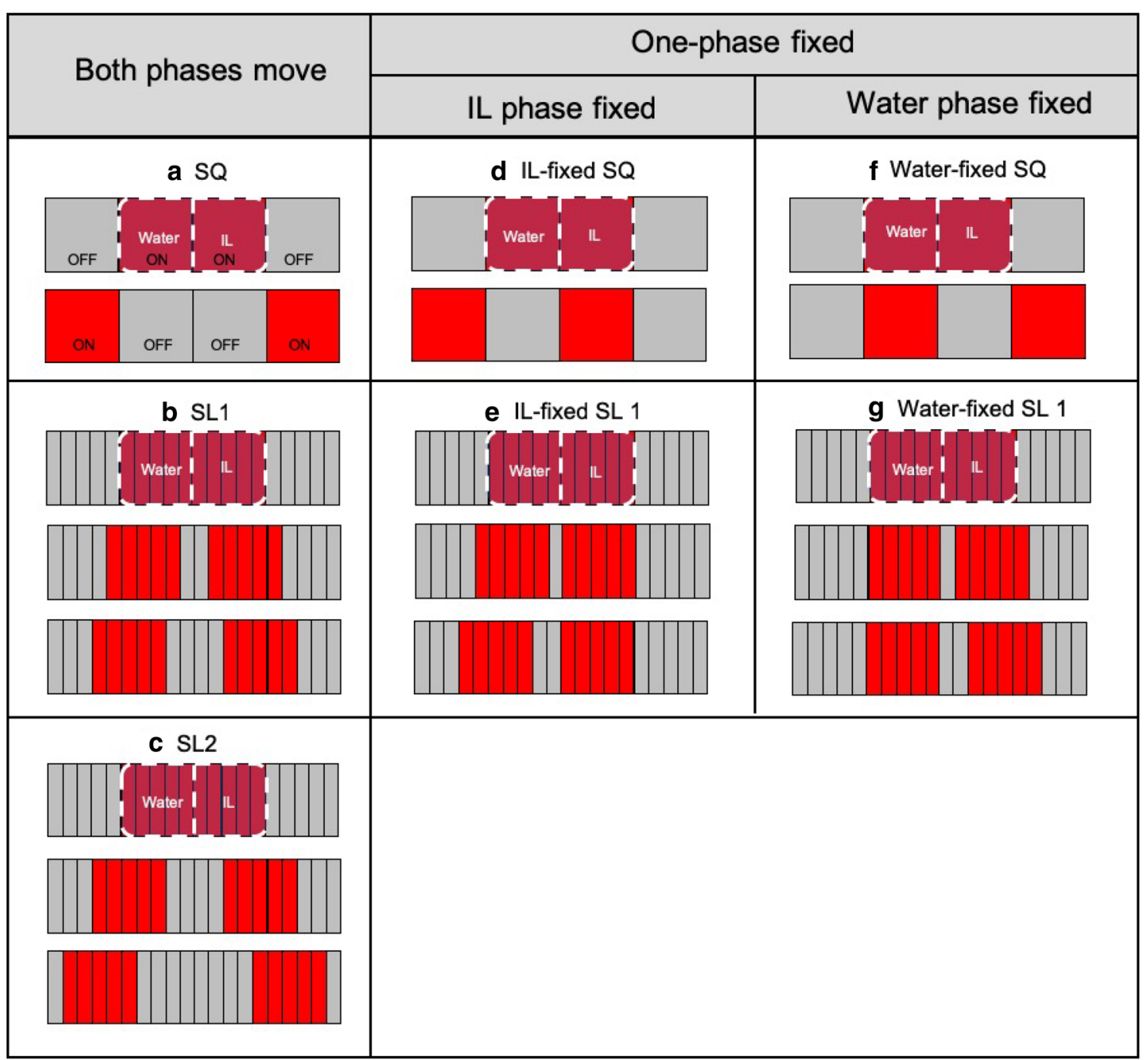

Fig. 7 List of actuation schemes tested in this study. The first row of each scheme illustrates the initial multiphase droplet placement. The activated electrodes are colored in red while the grounded electrodes are colored in gray

the amount of water residue to IL is similar to IL residue to water in SL1.

In summary, we have Fig. 10 to understand the effects of different schemes on residue formation. Each column represents the average value of the residue percentage from 12 tests for a particular scheme. As shown in the figure, the first three columns are for the cases in which both phases were pulled in opposite directions. As predicted earlier, SL1 resulted the least residue (1\%) followed by SL2 (2.7\%) and SQ had significantly larger amount of residue $(17 \%)$ than the others. It should be noted that, if we go from SL1 through SL2 to SQ, not only the residue but also the standard deviation increases. This means that by using SL schemes, droplet behaviors are more predictable and deterministic. Whereas, with SQ schemes, the uncertainty from hydrodynamic instability increased. The last four columns in Fig. 10 are data from tests with one phase fixed. The trends observed for the first three columns still hold true. That is using SL schemes (i.e., IL fixed-SL1 and Water fixed-SL1) is associated with less residue than using SQ schemes (IL fixed-SQ and Water fixed-SQ). In addition, there were prominent differences between IL fixed-SQ and Water fixed-SQ. However, the effect of having one phase fixed is not significant in SL schemes. As shown, both IL-fixed SL1 and Water fixedSL1 resulted in very similar amount of residue with SL1, which can be seen in comparison of Figs. 8b and 9c. This is because SL schemes are inherently very restrictive so that keeping one phase still does not make it significantly more restrictive. Whereas, in SQ scheme, forcing more 


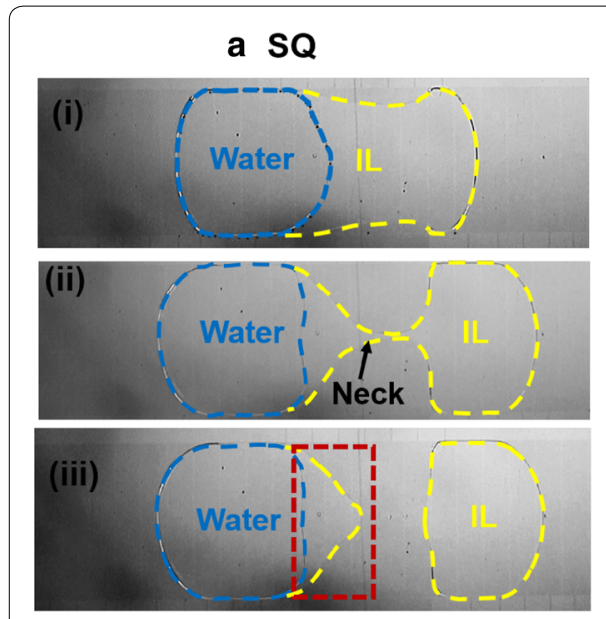

Residue b SL1
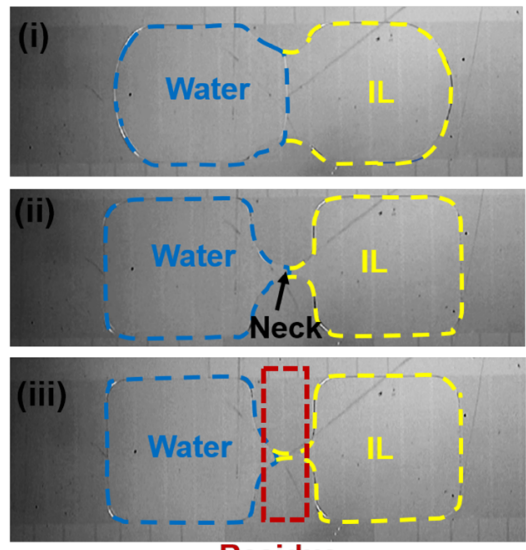

Residue b SL2
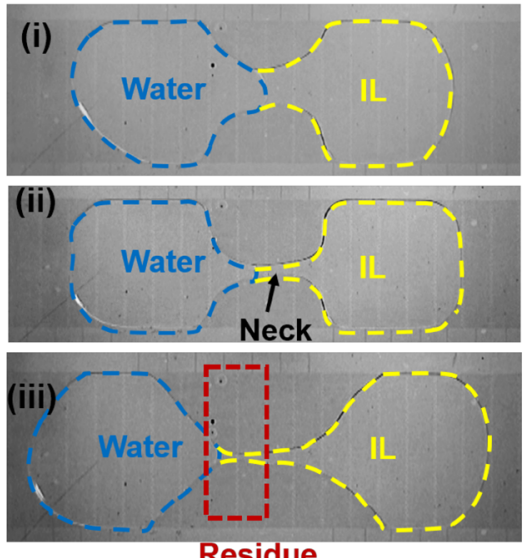

Residue

Fig. 8 Profiles of droplet deformation during the phase separation process. a SQ scheme, b SL1 scheme, and c SL2 scheme

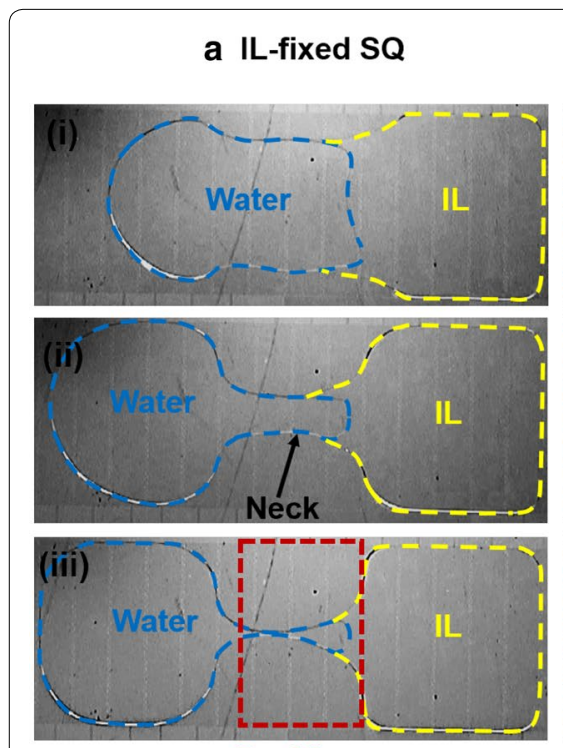

Residue
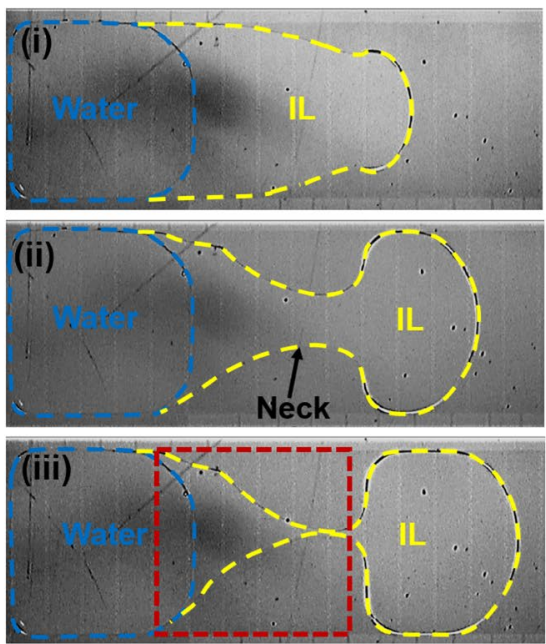

Residue c IL-fixed SL1
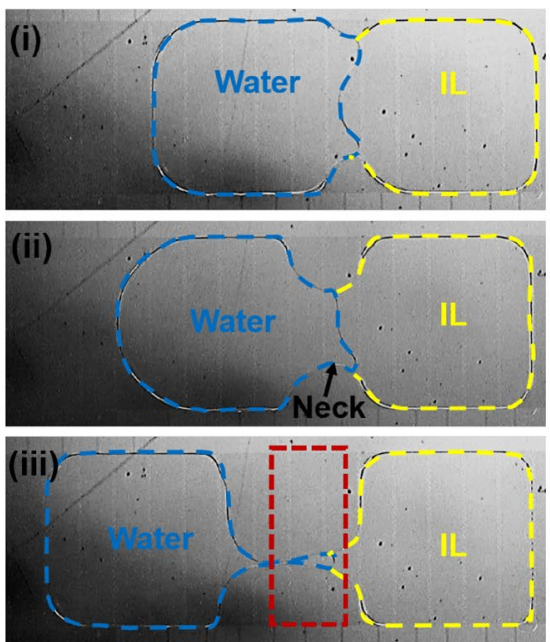

Residue

Fig. 9 Profiles of droplet deformation during the phase separation process. a IL-fixed SQ scheme, $\mathbf{b}$ water-fixed SQ scheme, and $\mathbf{c} I L-f i x e d$ SL1 scheme

deforming phase (i.e., IL) to be still effectively minimizes deformation which might be otherwise significant.

\section{Conclusions}

In order to reduce the residue formation during mechanically separating phases of a multiphase droplet, we proposed two strategies: (1) keeping capillary numbers of the two phases equal to each other, and (2) directly restricting the two phases to deform similarly. For the first method, we applied different voltages to phases according to their surface tension ratio. For the second method, we introduced different electrode operating schemes in which droplet deformation was restricted to vary for the two phases within an acceptable limit. Both methods resulted in the decrease of the 


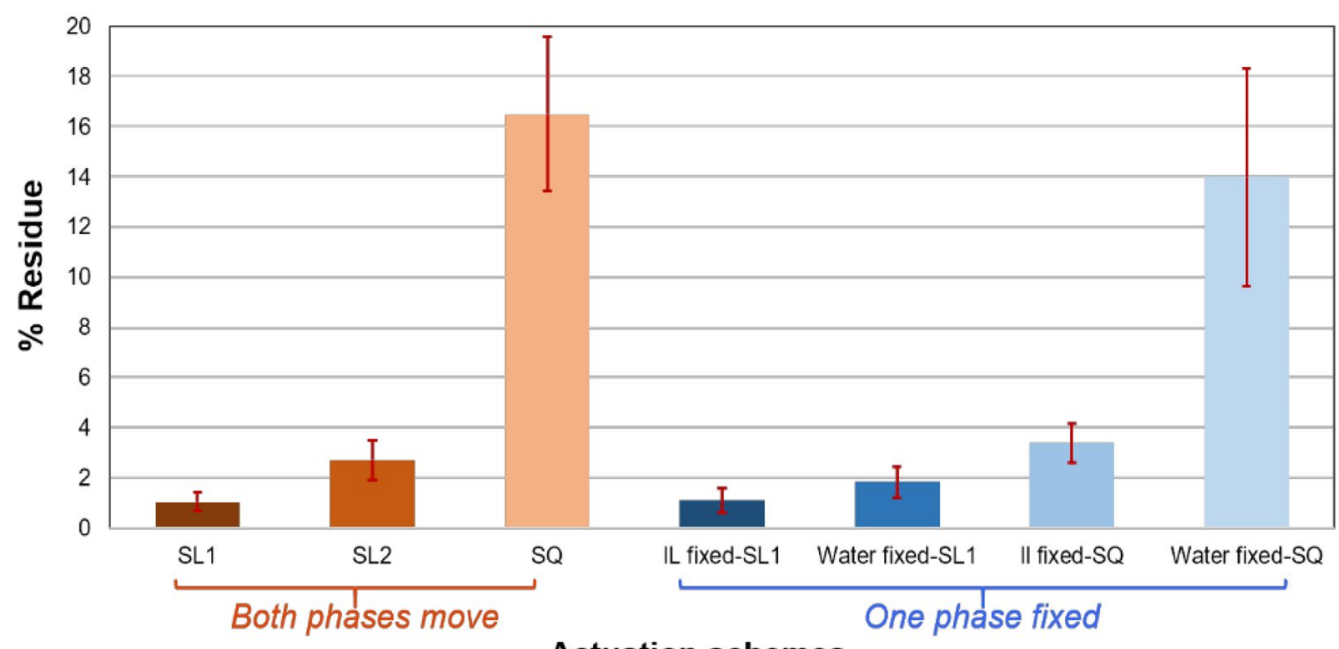

Fig. 10 Percent residue vs. schemes. Error bars represent standard deviations

residue after phase separation by $90 \%$ compared to the results from the conventional droplet splitting method using square electrodes and a single operation voltage.

\section{Supplementary information}

Supplementary information accompanies this paper at https://doi. org/10.1186/s40486-019-0099-0.

Additional file 1: S1. It contains description of device fabrication.

Additional file 2: S2. Video of separation using SL1.

Additional file 3: S3. Video of separation using SQ.

\section{Abbreviations}

EWOD: electrowetting on dielectric; DMF: digital microfluidics; ITO: indium tin oxide; SQ: device operation schemes using square electrodes; SL: device operation schemes using slender shape electrodes; IL: ionic liquid; Ca: capillary number.

\section{Acknowledgements}

Authors acknowledge the support by National Science Foundation (Award No. 1254602) for providing funding for this research.

\section{Authors' contributions}

The manuscript was written through contributions of all authors. Both authors read and approved the final manuscript.

\section{Funding}

National Science Foundation Award 1254602.

Availability of data and materials

Not applicable.

\section{Competing interests}

The authors declare that they have no competing interests.

Received: 1 August 2019 Accepted: 17 October 2019

Published online: 22 October 2019

\section{References}

1. LuY, Xia Y, Luo G (2011) Phase separation of parallel laminar flow for aqueous two phase systems in branched microchannel. Microfluid Nanofluid 10(5):1079-1086

2. Yamada M, Kasim V, Nakashima M, Edahiro Jl, Seki M (2004) Continuous cell partitioning using an aqueous two-phase flow system in microfluidic devices. Biotechnol Bioeng 88(4):489-494

3. Nam KH, Chang WJ, Hong H, Lim SM, Kim DI, Koo YM (2005) Continuous-flow fractionation of animal cells in microfluidic device using aqueous two-phase extraction. Biomed Microdevice 7(3):189-195

4. Münchow G, Hardt S, Kutter JP, Drese KS (2007) Electrophoretic partitioning of proteins in two-phase microflows. Lab Chip 7(1):98-102

5. Meagher RJ, Light YK, Singh AK (2008) Rapid, continuous purification of proteins in a microfluidic device using genetically-engineered partition tags. Lab Chip 8(4):527-532

6. Tsukamoto M, Taira S, Yamamura S, Morita Y, Nagatani N, Takamura Y, Tamiya E (2009) Cell separation by an aqueous two-phase system in a microfluidic device. Analyst. 134(10):1994-1998

7. Johansson HO, Ishii M, Minaguti M, Feitosa E, Penna TCV, Pessoa A Jr (2008) Separation and partitioning of green fluorescent protein from Escherichia coli homogenate in poly (ethylene glycol)/sodium-poly (acrylate) aqueous two-phase systems. Sep Purif Technol 62(1):166-174

8. Maruyama T, Matsushita H, Uchida JI, Kubota F, Kamiya N, Goto M (2004) Liquid membrane operations in a microfluidic device for selective separation of metal ions. Anal Chem 76(15):4495-4500

9. Reddy V, Zahn JD (2005) Interfacial stabilization of organic-aqueous two-phase microflows for a miniaturized DNA extraction module. J Colloid Interface Sci 286(1):158-165

10. Kashid MN, Harshe YM, Agar DW (2007) Liquid-liquid slug flow in a capillary: an alternative to suspended drop or film contactors. Ind Eng Chem Res 46(25):8420-8430

11. Zhao B, Moore JS, Beebe DJ (2001) Surface-directed liquid flow inside microchannels. Science 291(5506):1023-1026

12. Hibara A, Nonaka M, Hisamoto H, Uchiyama K, Kikutani Y, Tokeshi M, Kitamori T (2002) Stabilization of liquid interface and control of two-phase confluence and separation in glass microchips by utilizing octadecylsilane modification of microchannels. Anal Chem 74(7):1724-1728

13. Aota A, Nonaka M, Hibara A, Kitamori T (2007) Countercurrent laminar microflow for highly efficient solvent extraction. Angew Chem Int Ed 46(6):878-880 
14. Aota A, Mawatari K, Kitamori T (2009) Parallel multiphase microflows: fundamental physics, stabilization methods and applications. Lab Chip 9(17):2470-2476

15. Kikutani Y, Mawatari K, Hibara A, Kitamori T (2009) Circulation microchannel for liquid-liquid microextraction. Microchim Acta 164(3-4):241

16. Guzowski J, Korczyk PM, Jakiela S, Garstecki P (2012) The structure and stability of multiple micro-droplets. Soft Matter 8(27):7269-7278

17. Ma S, Huck WT, Balabani S (2015) Deformation of double emulsions under conditions of flow cytometry hydrodynamic focusing. Lab Chip 15(22):4291-4301

18. Li J, Chen H, Stone HA (2011) Breakup of double emulsion droplets in a tapered nozzle. Langmuir 27(8):4324-4327

19. Chen H, Li J, Shum HC, Stone HA, Weitz DA (2011) Breakup of double emulsions in constrictions. Soft Matter 7(6):2345-2347

20. Khan SA, Duraiswamy S (2009) Microfluidic emulsions with dynamic compound drops. Lab Chip 9(13):1840-1842

21. Lavrenteva OM, Rosenfeld L, Nir A (2011) Shape change, engulfment, and breakup of partially engulfed compound drops undergoing thermocapillary migration. Phys Rev E 84(5):056323

22. Neeson MJ, Tabor RF, Grieser F, Dagastine RR, Chan DY (2012) Compound sessile drops. Soft Matter 8(43):11042-11050

23. Stone HA (1994) Dynamics of drop deformation and breakup in viscous fluids. Annu Rev Fluid Mech 26(1):65-102

24. Pollack MG, Shenderov AD, Fair RB (2002) Electrowetting-based actuation of droplets for integrated microfluidics. Lab Chip 2:96

25. Cho SK, Moon H, Kim CJ (2003) Creating, splitting, cutting, and merging liquid droplets by electrowetting-based actuation for digital microfluidic circuits. J Microelectromech Syst 12:70-80

26. Mugele F, Baret JC (2005) Electrowetting: from basics to applications. J Phys Condens Matter 17:R705

27. Wijethunga PA, Nanayakkara YS, Kunchala P, Armstrong DW, Moon H (2011) On-chip drop-to-drop liquid microextraction coupled with realtime concentration monitoring technique. Anal Chem 83(5):1658-1664
28. Fan SK, Hsu YW, Chen CH (2011) Encapsulated droplets with metered and removable oil shells by electrowetting and dielectrophoresis. Lab Chip 11(15):2500-2508

29. Guan Y, Tong AY (2015) A numerical study of droplet splitting and merging in a parallel-plate electrowetting-on-dielectric device. J Heat Transf 137:091016

30. Nikapitiya NJ, Nahar MM, Moon H (2017) Accurate, consistent, and fast droplet splitting and dispensing in electrowetting on dielectric digital microfluidics. Micro Nano Syst Lett 5(1):24

31. Samiei E, Hoorfar M (2015) Systematic analysis of geometrical based unequal droplet splitting in digital microfluidics. J Micromech Microeng 25(5):055008

32. Banerjee A, Liu Y, Heikenfeld J, Papautsky I (2012) Deterministic splitting of fluid volumes in electrowetting microfluidics. Lab Chip 12(24):5138-5141

33. Baird ES, Mohseni K (2007) A unified velocity model for digital microfluidics. Nanoscale Microscale Thermophys Eng 11(1-2):109-120

34. Zulkepli SN, Hamid NH, Shukla V (2018) Droplet velocity measurement based on dielectric layer thickness variation using digital microfluidic devices. Biosensors. 8(2):45

35. Cui W, Zhang M, Duan X, Pang W, Zhang D, Zhang H (2015) Dynamics of electrowetting droplet motion in digital microfluidics systems: from dynamic saturation to device physics. Micromachines. 6(6):778-789

36. Nahar MM, Nikapitiya JB, You SM, Moon H (2016) Droplet velocity in an electrowetting on dielectric digital microfluidic device. Micromachines. $7(4): 71$

\section{Publisher's Note}

Springer Nature remains neutral with regard to jurisdictional claims in published maps and institutional affiliations.

\section{Submit your manuscript to a SpringerOpen ${ }^{\circ}$ journal and benefit from:}

- Convenient online submission

- Rigorous peer review

- Open access: articles freely available online

- High visibility within the field

- Retaining the copyright to your article

Submit your next manuscript at springeropen.com 